\title{
Do sleep-deprived adolescents make less-healthy food choices?
}

\author{
Allison K. Kruger ${ }^{1}$, Eric N. Reither ${ }^{2}$, Paul E. Peppard ${ }^{3}$, Patrick M. Krueger ${ }^{4}$ and Lauren Hale ${ }^{1 *}$ \\ ${ }^{1}$ Stony Brook University, Program in Public Health, Stony Brook, NY 11794-8338, USA \\ ${ }^{2}$ Utah State University, Logan, UT, USA \\ ${ }^{3}$ University of Wisconsin-Madison, Madison, WI, USA \\ ${ }^{4}$ University of Colorado, Denver, CO, USA \\ (Submitted 17 September 2013 - Final revision received 9 January 2014 - Accepted 10 January 2014 - First published online 13 February 2014)
}

\section{Abstract}

Short sleep duration among children and adolescents has been reported to be associated with elevated BMI and other adverse health outcomes. Food choices are one proposed mechanism through which this association may occur. In the present study, we examined whether self-reported habitual sleep duration is associated with vegetable and fruit consumption and fast food consumption. Using cross-sectional data from the National Longitudinal Study of Adolescent Health ( $n$ 13 284), we estimated three nested logistic regression models for two outcome variables: daily vegetable and fruit consumption and previous week's fast food consumption. The adjusted models included demographic and social/behavioural covariates. Self-reported habitual short sleep duration $(<7 \mathrm{~h} /$ night $)$ was associated with reduced odds of vegetable and fruit consumption compared with the recommended sleep duration $(>8 \mathrm{~h} / \mathrm{night})(\mathrm{OR} 0 \cdot 66, P<0 \cdot 001)$, even after adjusting for demographic and social/behavioural factors (OR $0.75, P<0.001)$. Short sleep duration was also associated with increased odds of fast food consumption (OR 1.40, $P<0 \cdot 001$ ) even after adjustment (OR 1.20, $P<0 \cdot 05$ ). Food choices are significantly associated with sleep duration and may play an important role in the mediation of the association between sleep and health among adolescents.

\section{Key words: Sleep: Sleep duration: Diet: Adolescents: Food choices}

Short sleep duration and poor sleep quality are associated with a wide range of negative health outcomes, including obesity, type 2 diabetes, heart disease and some cancers ${ }^{(1-10)}$. However, the mechanisms by which these associations occur are not well understood. One mechanism through which short sleep duration may be associated with these negative health outcomes is the regulation of energy balance ${ }^{(11)}$. Previous literature has found significant associations between sleep duration and leptin and ghrelin, two hormonal appetite regulators ${ }^{(12-14)}$. Therefore, changes in appetite due to changes in these hormones may affect dietary choices and decision-making, in terms of both the quality of food and the quantity of food consumed $^{(15-17)}$. For example, a recent study ${ }^{(18)}$ has found that acute sleep deprivation is associated with increased food purchasing in men. Finally, individuals who sleep less may have different lifestyles - e.g. more sedentary activities - that are associated with less-healthy food choices ${ }^{(19,20)}$.

Several studies have examined associations between sleep duration and diet among children and adolescents. These studies have reported relatively consistent findings across the observational studies ${ }^{(21-25)}$, but some inconsistencies in the smaller experimental studies ${ }^{(26-28)}$. For example, one observational study of over 3300 adolescents from ten European cities has found that the proportion of adolescents eating adequate amounts of fruit and vegetables is significantly lower among short-duration sleepers than among those who sleep $\geq 8 \mathrm{~h} /$ night $^{(21)}$. Another recent observational study of 240 American adolescents has found that those who sleep $<8 \mathrm{~h} /$ night consume a higher percentage of energy from fat than those who sleep $\geq 8 \mathrm{~h} /$ night $^{(22)}$. Similarly, a 2012 study of Iranian girls has found that short sleep duration is associated with poor diet quality, despite similar diet energy density across groups with differing sleep durations ${ }^{(25)}$.

Observational studies have also obtained similar results in even younger populations. For instance, one study of 10- and 11-year-old Finnish children has found that shorter sleep duration is associated with the consumption of more energy-rich foods and fewer nutrient-dense foods, with boys exhibiting a stronger association than girls ${ }^{(23)}$. Another study of Danish school children has found that sleep duration is negatively associated with dietary risk factors for obesity such as energy density, added sugars and consumption of sugar-sweetened beverages ${ }^{(24)}$.

In support of this observational research, two experimental studies have found that sleep restriction stimulates appetite and food consumption. One study amoung young men found an association between sleep deprivation and increased self-reported hunger and larger portion size, especially for snack foods ${ }^{(26)}$. Another study of both male and female adolescents found that chronic sleep restriction is associated with 
increased consumption of energy, carbohydrates, and foods with a high glycaemic index such as sweets and desserts ${ }^{(27)}$.

However, two studies call these results into question. A recent experimental study of twenty-one adolescent males has found that short-term sleep restriction ( $4 \mathrm{~d})$ is associated with decreased ad libitum energy intake and decreased motivation to eat ${ }^{(28)}$. In addition, one observational study of 550 Canadian school children has found that short sleep duration is independently associated with overweight and obesity, but that neither energy intake nor snacking mediates this association $^{(29)}$. However, this study does not have a representative sample, because participants had to have at least one obese biological parent.

To date, no USA-based nationally representative studies have investigated whether dietary choices vary by habitual sleep duration during adolescence. Adolescence represents a 'critical period for normal growth and development in which sleep... plays an important role ${ }^{\text {(30) }}$. Moreover, previous literature suggests that sleep and dietary habits formed in adolescence tend to persist into adulthood ${ }^{(31,32)}$, highlighting the importance of studying this crucial period of development. Consequently, the present study examined associations between sleep duration and both healthy and unhealthy food choices in a large nationally representative sample of American teenagers. We hypothesised that short sleep duration is associated with lesser consumption of healthy foods and greater consumption of unhealthy foods.

\section{Methods}

\section{Data}

Data analysed in the present study were from the National Longitudinal Study of Adolescent Health (Add Health), which has conducted in-home interviews in a nationally representative sample of American adolescents and young adults over the period 1994-2008. We used in-home interview data from Wave II, which were collected in 1996 from 14738 adolescent participants (88.6\% response rate). More details on study design are available online ${ }^{(33)}$. Wave II data were analysed, as Wave II was the only wave in which all participants were adolescents. Wave I included younger participants (some under 13 years old), while Waves III and IV examined the cohort in young adulthood and adulthood, respectively. Furthermore, Wave II was the only wave in which adolescents were asked questions about specific dietary choices, allowing us to collect information about fruit and vegetable consumption. The present analysis used the restricted-use dataset, resulting in 13284 adolescents with non-missing data.

\section{Measures}

Outcome variables - food choices. The main outcomes analysed include vegetable and fruit consumption and fast food consumption. The vegetable and fruit consumption variable was defined as whether or not the adolescent reported eating at least one vegetable and at least one fruit on the previous day. The interviewer prompted the adolescent participant as follows: 'Think about everything you had to eat and drink yesterday. This includes snacks as well as your regular meals.' The interviewer then asked a series of questions about specific food consumption on the previous day, such as 'Yesterday, did you eat cantaloupes, melons, mangoes, or papayas?' and 'Yesterday, did you eat string beans, green beans, peas, or snow peas?' The participants responded with a dichotomous yes/no or had the option to select 'Don't know'.

The fast food consumption variable was created from information about how often the adolescent ate fast food, classified as a dichotomous variable: eating fast food zero or one time in the last $7 \mathrm{~d}$ or eating fast food two or more times in the last $7 \mathrm{~d}$. More than $50 \%$ of the adolescents reported eating fast food two or more times in the last $7 \mathrm{~d}$, with only $15.9 \%$ of the sample reporting not eating fast food in the past week. We tested several different specifications of the fast food cut-off, and the results did not change significantly. We chose to present the 'two or more times' results because of the extremely high prevalence of adolescents reporting eating fast food one or more times per week.

Main explanatory variable - self-reported habitual sleep duration. The sleep duration variable was created from adolescents' self-reports of how many hours of sleep they usually get. Responses were organised into three categories in accordance with previous literature and recommendations from the American Academy of Pediatrics: short sleep duration ( $<7 \mathrm{~h} /$ night); mid-range sleep duration $(7-8 \mathrm{~h} /$ night); recommended sleep duration $(>8 \mathrm{~h} / \text { night })^{(34,35)}$

Covariates. Demographic covariates included sex, age (continuous), race/ethnicity (non-Hispanic white, non-Hispanic black, Hispanic, and all other races), and pubertal status ( 1 if the adolescents have reached puberty; 0 otherwise).

Social and behavioural covariates included family socioeconomic status, perception of neighbourhood safety, physical activity level, screen time, number of siblings at home, and presence of two biological parents at home. Family socioeconomic status variable was proxied by adolescents' reports of mother's level of education with three categories: less than high school; high-school diploma/General Educational Development (GED); some college or more. We used conditional mean imputation to predict mother's level of education for adolescents with missing values because 1502 participants (>10\% of the sample) had missing observations for this variable. Each imputed value was randomly drawn from the distribution of likely values, conditional on the observed covariates, so that that imputed values would better reflect the variability in the observed data ${ }^{(36)}$.

The perception of neighbourhood safety variable was created using adolescents' self-reports of feeling unsafe in their neighbourhood ( 1 if the adolescents reported feeling unsafe; 0 otherwise). Physical activity level was measured by how often the adolescents reported playing an active sport or exercising in the past week. Although the Centers for Disease Control recommends that adolescents participate in $1 \mathrm{~h}$ of physical activity every day ${ }^{(37)}$, only $33 \cdot 1 \%$ of the adolescents reported playing an active sport or exercising five or more times in the last $7 \mathrm{~d}$. Thus, the variable was divided into three categories: $0-1 ; 2-4 ; \geq 5$ times/week. 
Screen time was assessed as self-reported hours spent watching television and videos and playing video and computer games in the past week, divided into three categories: 0-14; 15-28; and $\geq 29 \mathrm{~h} /$ week. The low screen time category of $\leq 14 \mathrm{~h} /$ week was based on the Academy of Pediatrics guidelines for recommended screen time ${ }^{(38)}$.

Number of siblings at home, a continuous variable, was determined from adolescents' self-report about family members in the household. The biological parent variable was also determined from adolescents' self-report about family members at home ( 1 if the adolescents reported two biological parents at home; 0 otherwise).

\section{Statistical analyses}

All statistical analyses were conducted using Stata version 12.1 (Stata Corporation). We examined the prevalence of both healthy and unhealthy food choices among the short, midrange and recommended sleepers. We adjusted for complex, multistage sample design in all analyses using sample weights with svy commands. ANOVA and Pearson's $\chi^{2}$ tests were conducted to test for differences in food choices and covariates across the food choice categories. Logistic regression analysis was used to examine associations between food choices and sleep duration. For both food choice outcome variables, we used three nested models. Model 1 examined the association between food choices and sleep duration. Model 2 adjusted for demographic covariates (i.e. age, sex, race/ethnicity, and pubertal status). Model 3 additionally adjusted for social and behavioural covariates (i.e. mother's education level, perception of neighbourhood safety, physical activity level, screen time, number of siblings at home, and presence of biological parents at home). We tested for interactions between sleep duration and several sociodemographic variables, including sex, age, mother's education level, and race/ethnicity. We also conducted sensitivity analyses by categorising sleep duration and our food consumption variables into multiple different specifications (e.g. as a continuous variable and as various categorical cut points).

\section{Results}

\section{Characteristics of the study sample}

The mean age of adolescents in the study sample was 16 years (range: 11-22 years; $10-90$ th percentile: 14-18 years), and half the adolescents were male (49.9\%) (Table 1). NonHispanic white adolescents made up $67 \cdot 4 \%$ of the sample,

Table 1. Descriptive statistics of the sample by dietary choices $(n 13284) \dagger$

\begin{tabular}{|c|c|c|c|c|c|c|c|}
\hline & \multicolumn{3}{|c|}{ Fruit and vegetable consumption (\%)‡ } & \multicolumn{3}{|c|}{ Fast food consumption (\%)§ } & \multirow[b]{2}{*}{ Total } \\
\hline & Yes & No & $P$ & No & Yes & $P$ & \\
\hline \multicolumn{8}{|l|}{ Sleep duration } \\
\hline Short $(<7 \mathrm{~h})$ & $15 \cdot 1$ & $21 \cdot 6$ & $<0.0001^{* * *}$ & $15 \cdot 8$ & 19.5 & $0.0001^{\star \star \star}$ & $18 \cdot 0$ \\
\hline Mid-range $(7-8 h)$ & $62 \cdot 7$ & 57.4 & $<0.0001^{\star \star *}$ & 60.9 & $60 \cdot 0$ & 0.4941 & $60 \cdot 3$ \\
\hline Recommended (>8h) & $22 \cdot 2$ & $21 \cdot 0$ & 0.2402 & $23 \cdot 3$ & $20 \cdot 5$ & $0.0230^{\star}$ & $21 \cdot 7$ \\
\hline Mean age (years) & $15 \cdot 8$ & $16 \cdot 1$ & $<0.0001^{\star \star *}$ & $15 \cdot 7$ & $16 \cdot 1$ & $<0.0001^{\star \star *}$ & $16 \cdot 0$ \\
\hline \multicolumn{8}{|l|}{ Sex } \\
\hline Male & $49 \cdot 6$ & $50 \cdot 3$ & 0.6234 & $47 \cdot 3$ & $51 \cdot 8$ & $0.0003^{\star * \star}$ & 49.9 \\
\hline \multicolumn{8}{|l|}{ Race/ethnicity } \\
\hline White & 67.5 & $67 \cdot 3$ & 0.9007 & $67 \cdot 4$ & 67.4 & 0.9883 & $67 \cdot 4$ \\
\hline Black & $13 \cdot 0$ & $17 \cdot 8$ & $<0.0001^{\star \star \star}$ & 14.7 & $15 \cdot 5$ & 0.4840 & $15 \cdot 2$ \\
\hline Hispanic & $13 \cdot 4$ & $10 \cdot 7$ & $0.0034^{\star \star}$ & $12 \cdot 3$ & $12 \cdot 1$ & 0.8591 & $12 \cdot 2$ \\
\hline Other & $6 \cdot 1$ & $4 \cdot 1$ & $0.0011^{\star *}$ & $5 \cdot 6$ & $5 \cdot 0$ & 0.3470 & $5 \cdot 2$ \\
\hline \multicolumn{8}{|l|}{ Pubertal status } \\
\hline Reached puberty & 73.6 & 73.5 & 0.9681 & 73.7 & 73.5 & 0.8735 & 73.6 \\
\hline \multicolumn{8}{|l|}{ Mother's education level } \\
\hline Less than high school & $16 \cdot 1$ & $16 \cdot 7$ & 0.5622 & $16 \cdot 9$ & $16 \cdot 0$ & 0.2696 & $16 \cdot 4$ \\
\hline High-school diploma/GED & $38 \cdot 6$ & 43.7 & $0.0001^{* \star *}$ & $41 \cdot 3$ & $40 \cdot 5$ & 0.5046 & $40 \cdot 8$ \\
\hline Some college or more & $45 \cdot 3$ & $40 \cdot 0$ & $0.0001^{\star \star \star}$ & $42 \cdot 0$ & 43.5 & $0 \cdot 1960$ & $42 \cdot 8$ \\
\hline \multicolumn{8}{|l|}{ Perception of neighbourhood safety } \\
\hline Feel unsafe & $9 \cdot 5$ & $11 \cdot 1$ & 0.0549 & $10 \cdot 4$ & $10 \cdot 0$ & 0.5346 & $10 \cdot 2$ \\
\hline \multicolumn{8}{|l|}{ Physical activity level } \\
\hline Low (0-1 times/week) & $12 \cdot 5$ & 23.4 & $<0.0001^{\star \star \star}$ & $16 \cdot 1$ & $18 \cdot 1$ & $0.0336^{*}$ & $17 \cdot 3$ \\
\hline Medium (2-4 times/week) & $48 \cdot 1$ & $51 \cdot 5$ & $0.0056^{\star *}$ & $48 \cdot 5$ & $50 \cdot 4$ & 0.0739 & $49 \cdot 6$ \\
\hline High ( $\geq 5$ times/week) & $39 \cdot 4$ & $25 \cdot 1$ & $<0.0001^{\star \star *}$ & 35.4 & 31.4 & $0.0007^{\star \star \star}$ & 33.1 \\
\hline \multicolumn{8}{|l|}{ Screen time } \\
\hline Low (0-14h/week) & $50 \cdot 0$ & $46 \cdot 0$ & $0.0034^{\star \star}$ & $50 \cdot 5$ & $46 \cdot 4$ & $0.0048^{\star *}$ & $48 \cdot 2$ \\
\hline Medium (15-28 h/week) & $27 \cdot 9$ & $26 \cdot 8$ & 0.3222 & $27 \cdot 1$ & $27 \cdot 7$ & 0.6394 & $27 \cdot 4$ \\
\hline High ( $\geq 29 \mathrm{~h} /$ week) & $22 \cdot 2$ & $27 \cdot 2$ & $0.0001^{\star \star \star}$ & $22 \cdot 3$ & $25 \cdot 9$ & $0.0011^{\star \star}$ & 24.4 \\
\hline Mean number of siblings at home & 1.38 & 1.31 & $0.017^{\star}$ & 1.40 & 1.31 & $0.004^{\star \star}$ & 1.35 \\
\hline Presence of two biological parents at home & $56 \cdot 5$ & $48 \cdot 3$ & $<0.0001^{\star * \star}$ & 54.0 & $52 \cdot 1$ & $0 \cdot 1770$ & $52 \cdot 9$ \\
\hline
\end{tabular}

GED, General Educational Development.

Values were significantly different: ${ }^{\star} P<0.05$, ${ }^{\star *} P<0.01$, ${ }^{\star \star *} P<0.001$.

†Participants were excluded if they had missing data on any of the following variables: sleep duration; age; race/ethnicity. Due to the high prevalence of missing data on mother's education level, we imputed these values.

$\ddagger$ Yes $=$ consumed at least one fruit and vegetable on the previous day; No = did not consume at least one fruit and vegetable on the previous day.

$\S$ Yes $=$ consumed fast food $\geq 2$ times in the previous week; No = consumed fast food $0-1$ times in the previous week. 
with $15 \cdot 2 \%$ being non-Hispanic black adolescents, $12 \cdot 2 \%$ being Hispanic adolescents, and the remaining $5 \cdot 2 \%$ being adolescents of other races. A large percentage of the adolescents reported having reached puberty (73.6\%).

More than $80 \%$ of the adolescents reported that their mother had received at least a high-school diploma/GED, with $16.4 \%$ reporting their mother to be less educated. Few adolescents reported feeling unsafe in their neighbourhood $(10 \cdot 2 \%)$. The majority of adolescents reported engaging in physical activity at least 2 times/week, with $17 \cdot 3 \%$ reporting 0-1 times/week, $49.6 \%$ reporting 2-4 times/week, and $33.1 \%$ reporting $\geq 5$ times/week. Almost half of the sample (48.2\%) reported a non-excessive level of screen time, with $27 \cdot 4 \%$ viewing $15-28 \mathrm{~h} /$ week and $24.4 \%$ viewing $\geq 29 \mathrm{~h} /$ week. The mean number of siblings at home was 1.35 , and $52.9 \%$ of the adolescents reported having two biological parents living with them at home.

More than half of the adolescents (55.9\%) reported eating at least one vegetable and one fruit on the previous day, and the majority of adolescents (57.7\%) also reported consuming fast food two or more times in the last $7 \mathrm{~d}$.

Bivariate analyses showed that age, physical activity level, screen time, number of siblings at home, and sleep duration varied significantly by both fruit and vegetable consumption and fast food consumption (Table 1). Adolescents reporting unhealthy food choices (i.e. not consuming at least one fruit and one vegetable on the previous day and/or consuming fast food two or more times in the previous week) were significantly older and had significantly fewer siblings at home. Adolescents reporting high levels of physical activity consumed significantly greater amounts of fruit and vegetables and significantly less amounts of fast food. Greater screen time was associated with significantly less fruit and vegetable consumption and significantly greater fast food consumption. Finally, short-duration sleepers reported consuming significantly lower amounts of vegetables and fruit and significantly greater amounts of fast food.

Fast food consumption varied significantly by sex, with males reporting more frequent fast food consumption than females. Fruit and vegetable consumption varied significantly by race/ ethnicity, mother's education level, and presence of two biological parents at home. Black adolescents reported less fruit and vegetable consumption, while Hispanic adolescents and adolescents in the other race/ethnicity category reported significantly more fruit and vegetable consumption. Adolescents reporting mother's education level as high-school diploma/ GED were significantly less likely to report consuming fruit and vegetables, while those reporting mother's education

Table 2. OR of vegetable and fruit consumption

(Odds ratios and $95 \%$ confidence intervals; $n$ 13284)

\begin{tabular}{|c|c|c|c|c|c|c|}
\hline & \multicolumn{2}{|c|}{ Model 1} & \multicolumn{2}{|c|}{ Model $2 \dagger$} & \multicolumn{2}{|c|}{ Model 3ł } \\
\hline & OR & $95 \% \mathrm{Cl}$ & OR & $95 \% \mathrm{Cl}$ & OR & $95 \% \mathrm{Cl}$ \\
\hline \multicolumn{7}{|l|}{ Sleep duration } \\
\hline$<7 \mathrm{~h} /$ night & $0.66^{\star \star \star}$ & $0.57,0.76$ & $0.74^{\star \star \star}$ & $0.64,0.86$ & $0.75^{\star \star \star}$ & $0.64,0.88$ \\
\hline $7-8 \mathrm{~h} /$ night & 1.03 & $0.91,1.17$ & 1.08 & $0.95,1.24$ & 1.07 & $0.93,1.23$ \\
\hline$>8 \mathrm{~h} / \mathrm{night}$ & REF & & REF & & REF & \\
\hline Age (years) & & & $0.91^{* * *}$ & $0.88,0.94$ & $0.96^{*}$ & $0.93,0.99$ \\
\hline \multicolumn{7}{|l|}{ Sex } \\
\hline Male & & & 0.98 & $0.87,1 \cdot 10$ & $0.87^{\star \star}$ & $0.78,0.96$ \\
\hline Female & & & REF & & REF & \\
\hline \multicolumn{7}{|l|}{ Race/ethnicity } \\
\hline White & & & REF & & REF & \\
\hline Black & & & $0 \cdot 77^{\star \star \star}$ & $0.68,0.86$ & 0.89 & $0.79,1.01$ \\
\hline Hispanic & & & $1 \cdot 29^{\star \star}$ & $1.07,1.55$ & $1.43^{\star \star *}$ & $1.19,1.72$ \\
\hline Other race & & & $1.54^{\star \star \star}$ & $1.20,1.98$ & $1 \cdot 60^{\star \star \star}$ & $1.25,2.05$ \\
\hline Reached puberty $(=1)$ & & & 1.02 & $0.91,1.16$ & 1.00 & $0.88,1.13$ \\
\hline \multicolumn{7}{|l|}{ Mother's education level } \\
\hline Less than high school & & & & & 0.88 & $0.75,1.03$ \\
\hline High-school diploma/GED & & & & & $0.85^{\star \star}$ & $0.75,0.95$ \\
\hline Some college or more & & & & & REF & \\
\hline Perception of neighbourhood safety $(=1)$ & & & & & 0.90 & $0.76,1.07$ \\
\hline \multicolumn{7}{|l|}{ Physical activity level } \\
\hline Low (0-1 times/week) & & & & & REF & \\
\hline Medium (2-4 times/week) & & & & & $1 \cdot 70^{\star \star \star}$ & $1.49,1.94$ \\
\hline High ( $\geq 5$ times/week) & & & & & $2 \cdot 80^{\star * *}$ & $2 \cdot 39,3 \cdot 28$ \\
\hline \multicolumn{7}{|l|}{ Screen time } \\
\hline Low $(0-14 \mathrm{~h} /$ week $)$ & & & & & REF & \\
\hline Medium (15-28 h/week) & & & & & 0.91 & $0.81,1.02$ \\
\hline High ( $\geq 29 \mathrm{~h} /$ week $)$ & & & & & $0.77^{\star \star \star}$ & $0.66,0.89$ \\
\hline Number of siblings at home & & & & & 1.01 & $0.97,1.05$ \\
\hline Present of two biological parents at home & & & & & $1 \cdot 23^{\star * *}$ & $1.13,1.35$ \\
\hline
\end{tabular}

GED, General Educational Development.

REF, reference.

Values were significantly different: ${ }^{\star} P<0.05$, ${ }^{\star *} P<0.01,{ }^{\star \star *} P<0.001$.

† Model 2 adjusted for demographic covariates (i.e. age, sex, race/ethnicity, and pubertal status).

¥ Model 3 additionally adjusted for social/behavioural covariates (i.e. mother's education level, perception of neighbourhood safety, physical activity level, screen time, number of siblings at home, and presence of biological parents at home). 
level as some college or more were significantly more likely to report consuming fruit and vegetables. Finally, adolescents reporting two biological parents at home were significantly more likely to report consuming fruit and vegetables.

\section{Association between sleep duration and food choices}

Adolescents reporting short sleep duration $(<7 \mathrm{~h} /$ night) were less likely than those reporting the recommended sleep duration $(>8 \mathrm{~h} /$ night $)$ to consume at least one vegetable and one fruit on the previous day (model 1: OR 0.66, 95\% CI $0.57,0.76$ ) (Table 2). This association was significant after adjustment for demographic covariates (model 2: OR 0.74, $95 \%$ CI $0.64,0.86)$ as well as in the fully adjusted model, which also included social/behavioural covariates (model 3: OR $0.75,95 \%$ CI $0.64,0.88)$. Short-duration sleepers also were significantly more likely to report fast food consumption than the recommended-duration sleepers (model 1: OR 1.40, 95\% CI 1.18, 1.66) (Table 3). This association persisted in the model with demographic covariates (model 2: OR 1.20, $95 \%$ CI 1.01, 1.43) and also in the fully adjusted model (model 3: OR 1.20, $95 \%$ CI 1.01, 1.43).

Engaging in physical activity was associated with vegetable and fruit consumption, with adolescents reporting to be engaged in physical activity $\geq 5$ times/week significantly more likely to report consuming of vegetables and fruit than peers who exercised less (Table 2, OR 2.80, 95\% CI 2.39, 3.28). Physical activity was not significantly associated with the fast food consumption variable (Table 3).

Screen time exhibited a significant association with both food choice outcomes. For example, adolescents reporting high screen time activity ( $\geq 29 \mathrm{~h} /$ week) were $23 \%$ less likely than those reporting low screen time activity $(0-14 \mathrm{~h} /$ week $)$ to consume fruit and vegetables on the previous day in the fully adjusted model (Table 2, OR 0.77, $95 \%$ CI 0·66, 0.89). Adolescents reporting medium screen time activity (15-28h/week) and high screen time activity also had significantly higher odds of consuming fast food two or more times in the past week than those reporting low screen time activity (Table 3; medium: OR 1·17, $95 \%$ CI 1·03, 1·33; high: OR 1·34, $95 \%$ CI 1·18, 1.52).

Other covariates that exhibited statistically significant associations with vegetable and fruit consumption in the fully adjusted model were age, sex, Hispanic ethnicity, other race, mother's education level, and presence of two biological parents at home (Table 2). Similarly, age, sex, mother's education level, and number of siblings at home exhibited statistically significant associations with the fast food consumption variable in the fully adjusted model (Table 3).

Table 3. OR of fast food consumption

(Odds ratios and $95 \%$ confidence intervals; $n$ 13284)

\begin{tabular}{|c|c|c|c|c|c|c|}
\hline & \multicolumn{2}{|c|}{ Model 1} & \multicolumn{2}{|c|}{ Model $2 \dagger$} & \multicolumn{2}{|c|}{ Model $3 \ddagger$} \\
\hline & OR & $95 \% \mathrm{Cl}$ & OR & $95 \% \mathrm{Cl}$ & OR & $95 \% \mathrm{Cl}$ \\
\hline \multicolumn{7}{|l|}{ Sleep duration } \\
\hline$<7 \mathrm{~h} /$ night & $1.40^{\star \star *}$ & $1.18,1.66$ & $1 \cdot 20^{*}$ & $1.01,1.43$ & $1 \cdot 20^{*}$ & $1.01,1.43$ \\
\hline $7-8 \mathrm{~h} / \mathrm{night}$ & 1.12 & $0.97,1.29$ & 1.05 & $0.91,1.21$ & 1.05 & $0.91,1.21$ \\
\hline$>8 \mathrm{~h} / \mathrm{night}$ & REF & & REF & & REF & \\
\hline Age (years) & & & $1 \cdot 16^{\star \star *}$ & $1 \cdot 11,1 \cdot 20$ & $1 \cdot 16^{\star \star \star}$ & $1.12,1.21$ \\
\hline \multicolumn{7}{|l|}{ Sex } \\
\hline Male & & & $1 \cdot 20^{\star \star}$ & $1.07,1.34$ & $1 \cdot 17^{\star \star}$ & $1.05,1.30$ \\
\hline Female & & & REF & & REF & \\
\hline \multicolumn{7}{|l|}{ Race/ethnicity } \\
\hline White & & & REF & & REF & \\
\hline Black & & & 1.01 & $0.85,1.20$ & 0.97 & $0.82,1.16$ \\
\hline Hispanic & & & 0.96 & $0.82,1.12$ & 1.02 & $0.86,1.21$ \\
\hline Other race & & & 0.84 & $0.64,1.11$ & 0.85 & $0.65,1.12$ \\
\hline Reached puberty $(=1)$ & & & 1.03 & $0.91,1.17$ & 1.02 & $0.90,1.15$ \\
\hline \multicolumn{7}{|l|}{ Mother's education level } \\
\hline Less than high school & & & & & $0.84^{\star}$ & $0.72,0.97$ \\
\hline High-school diploma/GED & & & & & 0.91 & $0.81,1.02$ \\
\hline Some college or more & & & & & REF & \\
\hline Perception of neighbourhood safety $(=1)$ & & & & & 0.97 & $0.84,1.11$ \\
\hline \multicolumn{7}{|l|}{ Physical activity level } \\
\hline Low (0-1 times/week) & & & & & REF & \\
\hline Medium (2-4 times/week) & & & & & 1.01 & $0.89,1 \cdot 16$ \\
\hline High ( $\geq 5$ times/week) & & & & & 0.91 & $0.78,1.06$ \\
\hline \multicolumn{7}{|l|}{ Screen time } \\
\hline Low $(0-14 \mathrm{~h} /$ week $)$ & & & & & REF & \\
\hline Medium (15-28 h/week) & & & & & $1 \cdot 17^{\star}$ & $1.03,1.33$ \\
\hline High ( $\geq 29 \mathrm{~h} /$ week $)$ & & & & & $1.34^{\star \star \star}$ & $1.18,1.52$ \\
\hline Number of siblings at home & & & & & $0.95^{\star}$ & $0.91,1.00$ \\
\hline Presence of two biological parents at home & & & & & 0.99 & $0.88,1.11$ \\
\hline
\end{tabular}

REF, reference; GED, General Educational Development.

Values were significantly different: ${ }^{\star} P<0.05,{ }^{\star \star} P<0.01,{ }^{\star \star *} P<0.001$.

† Model 2 adjusted for demographic covariates (i.e. age, sex, race/ethnicity, and pubertal status).

$\ddagger$ Model 3 additionally adjusted for social/behavioural covariates (i.e. mother’s education level, perception of neighbourhood safety, physical activity level, screen time, number of siblings at home, and presence of biological parents at home). 
Our exploration of interaction terms between sleep duration and sociodemographic variables (sex, age, mother's education level, and race/ethnicity) led to a series of null findings, with one exception - a statistically significant interaction between sleep duration and Hispanic ethnicity. Specifically, relative to whites, Hispanic short-duration sleepers have much higher odds of consuming fast food $\geq 2$ times/week. However, based on the Wald test, the cluster of racial/ethnic interaction terms did not yield significant improvements in model fit, suggesting that model 3 is the best specification. Moreover, in additional analyses stratified by Hispanic ethnicity ( $n$ 2242), the increased odds of consuming fast food $\geq 2$ times/week did not reach statistical significance (OR $1.62, P=0.07)$. On balance, these results provide only weak and suggestive evidence that race/ethnicity modifies the effects of short sleep duration on dietary choices. As a final point, we report that sensitivity analyses with different specifications of the sleep duration variable yielded results similar to those of the above-described analyses.

\section{Discussion}

The present study found that short sleep duration $(<7 \mathrm{~h} /$ night) was associated with $25 \%$ decreased odds of adequate vegetable and fruit consumption and $20 \%$ increased odds of fast food consumption. These associations were robust to the inclusion of several important covariates. This suggests that sleep duration is independently associated with both healthy and unhealthy food choices per se and may also support the hypothesis that food choices may contribute to the association between sleep duration and obesity in American adolescents.

Interestingly, while the recommended sleep duration for adolescents is $>8 \mathrm{~h} /$ night, the analyses showed that mid-range sleepers $(7-8 \mathrm{~h} /$ night $)$ do not have significantly decreased odds of consuming vegetables and fruit or increased odds of consuming fast food compared with the recommendedduration sleepers. This suggests that the association of short sleep duration with dietary choices might occur only below a set threshold of habitual short sleep duration.

The present study used a large, nationally representative sample with a wide range of covariates to address the association between sleep duration and dietary choices in an adolescent sample. Although earlier studies ${ }^{(23-29,39-41)}$ have established significant associations between sleep duration and dietary choices, they were conducted in smaller non-representative samples. In addition, the present study examined both unhealthy and healthy food consumption variables, contributing to previous literature, whereas most studies have focused only on unhealthy dietary variables.

Although the present results are consistent with those of several observational studies ${ }^{(21-25)}$ and one small experimental study ${ }^{(27)}$, one experimental study has reported conflicting findings ${ }^{(28)}$. However, this study only explored short sleep duration over a short period of time $(4 \mathrm{~d})$ and failed to assess food intake and appetite after the four-night sleep restriction. Additionally, that study recruited only male participants and did not specifically allow for differential food choices, as the participants were only able to choose the quantity of food rather than the type of food.

Although self-reported sleep duration has been shown to be moderately associated with actigraphically assessed sleep duration on school nights data ${ }^{(42)}$, a more accurate measure of sleep, such as actigraphy, was not available at the time the present study was conducted, but should be considered for future population-based data collection efforts. In addition, the sleep data used fail to differentiate between weekday and weekend sleep, which may be relevant when examining dietary choices. Furthermore, the Add Health in-home interview questions regarding food consumption, although detailed, fail to provide specific information about quantity and timing. The quantity of food consumed is especially important, as it would allow us to control for total energy intake, which has been hypothesised in previous literature to mediate the association between sleep duration and obesity ${ }^{(43,44)}$.

The present study demonstrates that sleep duration may be related to both healthy and unhealthy food choices of adolescents. The next step is to investigate the causal mechanisms behind the association between sleep duration and energy balance. For example, St-Onge \& Shechter ${ }^{(45)}$ point out that the timing of sleep may also play a role in dietary patterns. Understanding the causal mechanisms may lead to the development of programmes that aim to improve sleep and sleep hygiene as an important and underappreciated component of health-promotion and obesity-prevention interventions.

\section{Acknowledgements}

The present study was supported by the National Institute of Diabetes and Digestive and Kidney Diseases (R21DK089414) and received administrative support from the National Institute of Child Health and Human Development (grant R24 HD066613) at the National Institutes of Health.

The authors' contributions are as follows: L. H. conceived the project; A. K. K. and L. H. conducted the data analyses and drafted the manuscript; E. N. R., P. M. K. and P. E. P. assisted with data interpretation, imputation, sensitivity testing of the data analyses and manuscript revisions.

None of the authors has any conflicts of interest to declare.

\section{References}

1. Gottlieb DJ, Redline S, Nieto FJ, et al. (2006) Association of usual sleep duration with hypertension: the Sleep Heart Health Study. Sleep 29, 1009-1014.

2. Newman AB, Enright PL, Manolio TA, et al. (1997) Sleep disturbance, psychosocial correlates, and cardiovascular disease in 5201 older adults: the Cardiovascular Health Study. J Am Geriatr Soc 45, 1-7.

3. Pack AI, Maislin G, Staley B, et al. (2006) Impaired performance in commercial drivers: role of sleep apnea and short sleep duration. Am J Respir Crit Care Med 174, 446-454.

4. Connor J, Norton R, Ameratunga S, et al. (2002) Driver sleepiness and risk of serious injury to car occupants: population based case control study. BMJ 324, 1125.

5. Lockley SW, Cronin JW, Evans EE, et al. (2004) Effect of reducing interns' weekly work hours on sleep and attentional failures. New Engl J Med 351, 1829-1837. 
6. Cappuccio FP, Taggart FM, Kandala NB, et al. (2008) Metaanalysis of short sleep duration and obesity in children and adults. Sleep 31, 619-626.

7. Liu J, Zhang A \& Li L (2012) Sleep duration and overweight/ obesity in children: review and implications for pediatric nursing. J Spec Pediatr Nurs 17, 193-204.

8. Gupta NK, Mueller WH, Chan W, et al. (2002) Is obesity associated with poor sleep quality in adolescents? $\mathrm{Am} \mathrm{J}$ Hum Biol 14, 762-768.

9. Hasler G, Buysse DJ, Klaghofer R, et al. (2004) The association between short sleep duration and obesity in young adults: a 13-year prospective study. Sleep 27, 661-666.

10. von Kries R, Toschke AM, Wurmser H, et al. (2002) Reduced risk for overweight and obesity in 5- and 6-y-old children by duration of sleep - a cross-sectional study. Int J Obes Relat Metab Disord 26, 710-716.

11. St-Onge MP (2013) The role of sleep duration in the regulation of energy balance: effects on energy intakes and expenditure. J Clin Sleep Med 9, 73-80.

12. Taheri S, Lin L, Austin D, et al. (2004) Short sleep duration is associated with reduced leptin, elevated ghrelin, and increased body mass index. PLoS Med 1, e62.

13. Spiegel K, Tasali E, Penev P, et al. (2004) Brief communication: sleep curtailment in healthy young men is associated with decreased leptin levels, elevated ghrelin levels, and increased hunger and appetite. Ann Intern Med 141, 846-850.

14. St-Onge MP, O'Keeffe M, Roberts AL, et al. (2012) Short sleep duration, glucose dysregulation and hormonal regulation of appetite in men and women. Sleep 35, 1503-1510.

15. Leibel RL (2002) The role of leptin in the control of body weight. Nutr Rev 60, S15-S19, discussion S68-S84, 5-7.

16. van der Lely AJ, Tschop M, Heiman ML, et al. (2004) Biological, physiological, pathophysiological, and pharmacological aspects of ghrelin. Endocr Rev 25, 426-457.

17. St-Onge MP, McReynolds A, Trivedi ZB, et al. (2012) Sleep restriction leads to increased activation of brain regions sensitive to food stimuli. Am J Clin Nutr 95, 818-824.

18. Chapman CD, Nilsson EK, Nilsson VC, et al. (2013) Acute sleep deprivation increases food purchasing in men. Obesity (Silver Spring) 21, E555-E560.

19. Olds T, Ridley K \& Dollman J (2006) Screenieboppers and extreme screenies: the place of screen time in the time budgets of 10-13 year-old Australian children. Aust $N Z J$ Public Health 30, 137-142.

20. Nelson MC \& Gordon-Larsen P (2006) Physical activity and sedentary behavior patterns are associated with selected adolescent health risk behaviors. Pediatrics 117, 1281-1290.

21. Garaulet MOF, Ruiz JR, Rey-Lopez JP, et al. (2011) Short sleep duration is associated with increased obesity markers in European adolescents: effect of physical activity and dietary habits. The HELENA study. Int J Obes 35, 1308-1317.

22. Weiss A, Xu F, Storfer-Isser A, et al. (2010) The association of sleep duration with adolescents' fat and carbohydrate consumption. Sleep 33, 1201-1209.

23. Westerlund L, Ray C \& Roos E (2009) Associations between sleeping habits and food consumption patterns among 10-11-year-old children in Finland. Br J Nutr 102, 1531-1537.

24. Kjeldsen JS, Hjorth MF, Andersen R, et al. (2014) Short sleep duration and large variability in sleep duration are independently associated with dietary risk factors for obesity in Danish school children. Int J Obes (Lond) 38, 32-39.

25. Haghighatdoost F, Karimi G, Esmaillzadeh A, et al. (2012) Sleep deprivation is associated with lower diet quality indices and higher rate of general and central obesity among young female students in Iran. Nutrition 28, 1146-1150.
26. Hogenkamp PS, Nilsson E, Nilsson VC, et al. (2013) Acute sleep deprivation increases portion size and affects food choice in young men. Psychoneuroendocrinology 38, $1668-1674$.

27. Beebe DW, Simon S, Summer S, et al. (2013) Dietary intake following experimentally restricted sleep in adolescents. Sleep 36, 827-834.

28. Klingenberg L, Chaput JP, Holmback U, et al. (2012) Sleep restriction is not associated with a positive energy balance in adolescent boys. Am J Clin Nutr 96, 240-248.

29. Chaput JP, Lambert M, Gray-Donald K, et al. (2011) Short sleep duration is independently associated with overweight and obesity in Quebec children. Can J Public Health 102, 369-374.

30. Spear LP (2000) The adolescent brain and age-related behavioral manifestations. Neurosci Biobehav Rev 24, 417-463.

31. Lund HG, Reider BD, Whiting AB, et al. (2010) Sleep patterns and predictors of disturbed sleep in a large population of college students. $J$ Adolesc Health 46, 124-132.

32. Lake AA, Mathers JC, Rugg-Gunn AJ, et al. (2006) Longitudinal change in food habits between adolescence (11-12 years) and adulthood (32-33 years): the ASH30 Study. J Public Health (Oxf) 28, 10-16.

33. Harris KM, Halpern CT, Whitsel E, et al. (2009) The National Longitudinal Study of Adolescent Health: research design. http://www.cpc.unc.edu/projects/addhealth/design/

34. Mercer PW, Merritt SL \& Cowell JM (1998) Differences in reported sleep need among adolescents. $J$ Adolesc Health 23, 259-263.

35. Millman RP (2005) Excessive sleepiness in adolescents and young adults: causes, consequences, and treatment strategies. Pediatrics 115, 1774-1786.

36. Gelman A \& Hill J (2007) Data Analysis using Regression and Multilevel/Hierarchical Models. Cambridge/New York: Cambridge University Press.

37. US Department of Health and Human Services (2008) Physical activity guidelines for Americans. Washington, DC: US Department of Health and Human Services. http:// www.health.gov/paguidelines/pdf/paguide.pdf.

38. American Academy of Pediatrics \& Committee on Public Education (2001) American Academy of Pediatrics: children, adolescents, and television. Pediatrics 107, 423-426.

39. Nishiura CNJ \& Hashimoto H (2010) Dietary patterns only partially explain the effect of short sleep duration on the incidence of obesity. Sleep 33, 753-757.

40. Buxton OM, Quintiliani LM, Yang MH, et al. (2009) Association of sleep adequacy with more healthful food choices and positive workplace experiences among motor freight workers. Am J Public Health 99, S636-S643.

41. Tatone-Tokuda F, Dubois L, Ramsay T, et al. (2011) Sex differences in the association between sleep duration, diet and body mass index: a birth cohort study. J Sleep Res $\mathbf{2 1}$, 448-460.

42. Wolfson AR, Carskadon MA, Acebo C, et al. (2003) Evidence for the validity of a sleep habits survey for adolescents. Sleep 26, 213-216.

43. Nedeltcheva AV, Kilkus JM \& Imperial J (2009) Sleep curtailment is accompanied by increased intake of calories from snacks. Am J Clin Nutr 89, 126-133.

44. Patel SR, Malhotra A, White DP, et al. (2006) Association between reduced sleep and weight gain in women. $\mathrm{Am} \mathrm{J}$ Epidemiol 164, 947-954.

45. St-Onge MP \& Shechter A (2013) Sleep restriction in adolescents: forging the path towards obesity and diabetes? Sleep 36, 813-814. 